\title{
Nevirapine versus Ritonavir-Boosted Lopinavir for HIV-Infected Children
}

\author{
Avy Violari, F.C.Paed., Jane C. Lindsey, Sc.D., Michael D. Hughes, Ph.D., \\ Hilda A. Mujuru, M.D., Linda Barlow-Mosha, M.D., Portia Kamthunzi, M.D., \\ Benjamin H. Chi, M.D., Mark F. Cotton, M.Med., Harry Moultrie, M.D., \\ Sandhya Khadse, M.D., Werner Schimana, M.D., Raziya Bobat, M.D., \\ Lynette Purdue, Pharm.D., Susan H. Eshleman, M.D., Ph.D., Elaine J. Abrams, M.D., \\ Linda Millar, B.A., Elizabeth Petzold, Ph.D., Lynne M. Mofenson, M.D., \\ Patrick Jean-Philippe, M.D., and Paul Palumbo, M.D.
}

The authors' affiliations are listed in the Appendix. Address reprint requests to Dr. Palumbo at the International Pediatric HIV Program, Dartmouth-Hitchcock Medical Center, 1 Medical Center Dr., Lebanon, NH 03756, or at paul.e.palumbo@ dartmouth.edu.

N Engl J Med 2012;366:2380-9. Coppright $(2012$ Massachusetts Medical Society.

\section{ABSTRACT}

\section{BACKGROUND}

Nevirapine-based antiretroviral therapy is the predominant (and often the only) regimen available for children in resource-limited settings. Nevirapine resistance after exposure to the drug for prevention of maternal-to-child human immunodeficiency virus (HIV) transmission is common, a problem that has led to the recommendation of ritonavir-boosted lopinavir in such settings. Regardless of whether there has been prior exposure to nevirapine, the performance of nevirapine versus ritonavir-boosted lopinavir in young children has not been rigorously established.

\section{METHODS}

In a randomized trial conducted in six African countries and India, we compared the initiation of HIV treatment with zidovudine, lamivudine, and either nevirapine or ritonavir-boosted lopinavir in HIV-infected children 2 to 36 months of age who had no prior exposure to nevirapine. The primary end point was virologic failure or discontinuation of treatment by study week 24 .

\section{RESULTS}

A total of 288 children were enrolled; the median percentage of CD4+ T cells was $15 \%$, and the median plasma HIV type 1 (HIV-1) RNA level was $5.7 \log _{10}$ copies per milliliter. The percentage of children who reached the primary end point was significantly higher in the nevirapine group than in the ritonavir-boosted lopinavir group $(40.8 \%$ vs. $19.3 \%$; $\mathrm{P}<0.001)$. Among the nevirapine-treated children with virologic failure for whom data on resistance were available, more than half (19 of 32) had resistance at the time of virologic failure. In addition, the time to a protocoldefined toxicity end point was shorter in the nevirapine group $(\mathrm{P}=0.04)$, as was the time to death $(\mathrm{P}=0.06)$.

\section{CONCLUSIONS}

Outcomes were superior with ritonavir-boosted lopinavir among young children with no prior exposure to nevirapine. Factors that may have contributed to the suboptimal results with nevirapine include elevated viral load at baseline, selection for nevirapine resistance, background regimen of nucleoside reverse-transcriptase inhibitors, and the standard ramp-up dosing strategy. The results of this trial present policymakers with difficult choices. (Funded by the National Institute of Allergy and Infectious Diseases and others; P1060 ClinicalTrials.gov number, NCT00307151.) 
$\mathrm{N}$ EW ANTIRETROVIRAL DRUGS AND DRUG classes have markedly advanced the treatment of human immunodeficiency virus (HIV) infection in children. In resource-limited settings, where most HIV-infected children live, therapeutic options are restricted by financial and logistic constraints. Nevirapine is an important component of long-term therapy because it is stable at high temperatures, available in fixeddose combinations, and relatively inexpensive, and its use is based on extensive experience and an acceptable safety profile in the pediatric population. Often, it is the only option available for infants and children.

Randomized studies have shown that regimens incorporating ritonavir-boosted lopinavir (both protease inhibitors) are superior to nevirapine-based regimens in the treatment of mothers and infants who were previously exposed to single-dose nevirapine for the prevention of perinatal HIV transmission. ${ }^{1,2}$ The World Health Organization (WHO) guidelines for antiretroviral treatment (ART) now recommend ritonavirboosted lopinavir for the initial treatment of children younger than 2 years of age who have previously been exposed to single-dose nevirapine. ${ }^{3}$ Nevirapine continues to be recommended for initial therapy in children without prior exposure to nevirapine; however, there has been no randomized trial comparing ritonavir-boosted lopinavir-based ART and nevirapine-based ART in such children.

The P1060 study was designed as two parallel, randomized clinical trials comparing nevirapine with ritonavir-boosted lopinavir, in addition to zidovudine and lamivudine, in HIV-infected, ARTeligible children between 2 and 36 months of age. We previously published the findings in cohort 1 of the study, which comprised children with documented exposure to single-dose nevirapine. ${ }^{1}$ Here, we report the findings in cohort 2, which comprised children without previous nevirapine exposure.

\section{METHODS}

\section{PATIENT POPULATION AND STUDY DESIGN}

Children with HIV infection were eligible for the study if they had not previously been exposed to antiretroviral agents (except for those used to prevent mother-to-child transmission of HIV), if they required treatment according to WHO criteria, and if their baseline level of plasma HIV type 1
(HIV-1) RNA was above 5000 copies per milliliter. Children (and their mothers) could not have had previous exposure to non-nucleoside reversetranscriptase inhibitors (NNRTIs) such as nevirapine or efavirenz (hereinafter referred to as an absence of exposure to nevirapine).

The children were stratified by age ( 2 to $<6$, 6 to $<12$, or 12 to 36 months) and randomly assigned in equal numbers to either nevirapine or ritonavir-boosted lopinavir, combined with zidovudine and lamivudine. Nevirapine was initially given in a dose of $4 \mathrm{mg}$ per kilogram of body weight once daily for 14 days, with a dose of $7 \mathrm{mg}$ per kilogram twice daily thereafter (the dose approved by the Food and Drug Administration). An amendment to the protocol (September $4,2007)$ increased the nevirapine dose to 160 to $200 \mathrm{mg}$ per square meter of body-surface area (once daily for 14 days, then twice daily) in line with newly instituted WHO recommendations.

Children were enrolled at one site in India and at nine sites across sub-Saharan Africa (four in South Africa and one each in Zimbabwe, Zambia, Malawi, Uganda, and Tanzania). The study was approved by the ethics review committee at each site, the Ministries of Health (where appropriate), and the institutional review board at each partner institution in the United States. Each child's parent or legal guardian provided written informed consent. Study visits and laboratory testing were conducted as previously described. ${ }^{1}$

All the authors vouch for the completeness and accuracy of the data presented. The study was conducted in accordance with the protocol, available with the full text of this article at NEJM.org. The antirectroviral drugs used in this study were donated by Abbott, Boehringer Ingelheim, and GlaxoSmithKline. Representatives of these three pharmaceutical manufacturers participated in early discussions of the trial design but not in final design decisions or in trial implementation or analyses.

\section{STUDY END POINTS}

The primary study end point was treatment failure by 24 weeks, defined as virologic failure or permanent discontinuation of the nevirapine or ritonavir-boosted lopinavir component of the treatment regimen for any reason (concomitant tuberculosis therapy, death, or another reason). Virologic failure was defined as a confirmed plasma HIV-1 RNA level that was less than $1 \log _{10}$ 
copies per milliliter below the baseline level at 12 to 24 weeks after treatment initiation, or a confirmed plasma HIV-1 RNA level of more than 400 copies per milliliter at 24 weeks. Toxicity end points are defined in the protocol.

Secondary end points included confirmed virologic failure or death by week 24 , confirmed virologic failure (with virologic failure defined as above or as a confirmed viral rebound to $>4000$ copies per milliliter after week 24) or death during the follow-up period, and a composite of virologic failure or discontinuation of the study treatment during the follow-up period. Data for children lost to follow-up were censored for the virologic failure end point at the date of the last available HIV-1 RNA measurement.

\section{STATISTICAL ANALYSIS}

The analysis included all children in cohort 2 who started the study treatment. Four children who had previously been exposed to nevirapine were incorrectly assigned to cohort 2 . These four children were included in all analyses; sensitivity analyses that excluded them showed similar results. Since only four infants were enrolled in the youngest age stratum ( 2 to $<6$ months), children in the two youngest strata were combined for all the analyses.

Rates of the primary end point and of virologic failure at week 24 were calculated from Kaplan-Meier curves for each age stratum. The primary analysis was based on a weighted average of rates across age strata, with weights equal to the inverse of the variance of the stratumspecific rate. Unweighted rates (i.e., with age stratification ignored) were also evaluated. Agestratified Cox proportional-hazards models were used to compare time-to-event end points (in adjusted and unadjusted analyses) and to evaluate whether differences between randomly assigned treatments varied across subgroups by including a treatment-by-subgroup interaction variable. Prespecified subgroups were classified according to age, sex, initial dose of nevirapine, HIV subtype, and type of documentation supporting the absence of prior exposure to nevirapine, as well as baseline HIV-1 RNA level, percentage of CD4+ T cells, CD4+ count, and WHO disease stage.

Safety information was reported while the children were receiving the study treatment (astreated analysis). Summaries of adverse events according to the highest grade for each type of event were tabulated. Changes from baseline to weeks 24 and 48 in cholesterol levels, triglyceride levels, CD4+ counts and percentages, Centers for Disease Control and Prevention z scores for height and weight (www.cdc.gov/growthcharts), and body-mass index (BMI) were compared according to treatment with the use of t-tests (unadjusted) and linear regression analyses adjusted for entry value. Intention-to-treat analyses were performed for immunologic and growth outcomes, and as-treated analyses were performed for lipid data. Two-sided P values, unadjusted for interim analyses or multiple comparisons, are reported.

\section{RESULTS}

STUDY PARTICIPANTS, FOLLOW-UP, AND TREATMENT ADHERENCE

A total of 288 children were enrolled between November 23, 2006, and March 19, 2010, with follow-up planned to continue until March 2011. On October 27, 2010, when the 24-week followup for all children was complete, the data and safety monitoring board performed a review and recommended unblinding of the data and release of the results, since the comparisons of the two treatments for the primary end point had met prespecified stopping guidelines.

Reported analyses are based on data collected through October 27, 2010, for the 147 children randomly assigned to nevirapine and the 140 assigned to ritonavir-boosted lopinavir; 1 child in the ritonavir-boosted lopinavir group never started therapy and was therefore not included (see Table S1 in the Supplementary Appendix, available at NEJM.org). The median follow-up period was 72 weeks (interquartile range, 48 to 120). At baseline, 210 children (73.2\%) were 12 months of age or older, with a median age of 1.7 years (Table 1). The children had relatively advanced HIV disease, with a median HIV-1 RNA level of 535,632 copies per milliliter, a median of $15 \%$ CD4+ T cells, and median $z$ scores of -2.6 for weight and -2.3 for height. The majority of HIV infections (212 of 265 [80.0\%]) were subtype C. Median adherence to therapy was $100 \%$ for the initial 24 weeks of treatment, according to reports by caregivers, with no significant differences between the two treatment groups $(\mathrm{P}=0.16)$. Caregivers reported adherence of $95 \%$ or higher for $80.3 \%$ of the children who received nevirapine, as compared with $90.0 \%$ of those who received ritonavir-boosted lopinavir. 


\begin{tabular}{|c|c|c|c|c|c|c|c|}
\hline \multirow[t]{2}{*}{ Characteristic } & \multicolumn{3}{|c|}{$\begin{array}{l}\text { Nevirapine Group } \\
(\mathrm{N}=147)\end{array}$} & \multicolumn{3}{|c|}{$\begin{array}{l}\text { Ritonavir-Boosted Lopinavir Group } \\
\qquad(N=140)\end{array}$} & \multirow[t]{2}{*}{$\begin{array}{c}\text { Total } \\
(\mathrm{N}=287)\end{array}$} \\
\hline & $\begin{array}{c}\text { Age }<12 \mathrm{Mo} \\
(\mathrm{N}=41)\end{array}$ & $\begin{array}{c}\text { Age } \geq 12 \mathrm{Mo} \\
(\mathrm{N}=106)\end{array}$ & $\begin{array}{c}\text { All } \\
(\mathrm{N}=147)\end{array}$ & $\begin{array}{c}\text { Age }<12 \mathrm{Mo} \\
(\mathrm{N}=36)\end{array}$ & $\begin{array}{c}\text { Age } \geq 12 \mathrm{Mo} \\
(\mathrm{N}=104)\end{array}$ & $\begin{array}{c}\text { All } \\
(N=140)\end{array}$ & \\
\hline \multicolumn{8}{|l|}{ Age $-y r$} \\
\hline Median & 0.7 & 2.1 & 1.8 & 0.7 & 1.9 & 1.7 & 1.7 \\
\hline 10th-90th percentile & 0.5 to 1.0 & 1.3 to 2.9 & 0.7 to 2.9 & 0.5 to 0.9 & 1.3 to 2.8 & 0.6 to 2.7 & 0.6 to 2.8 \\
\hline Male sex - no. (\%) & $23(56.1)$ & $46(43.4)$ & $69(46.9)$ & $19(52.8)$ & $49(47.1)$ & $68(48.6)$ & $137(47.7)$ \\
\hline Breast-fed - no. (\%) & $30(73.2)$ & $88(83.0)$ & $118(80.3)$ & 33 (91.7) & $82(78.8)$ & $115(82.1)$ & $233(81.2)$ \\
\hline \multicolumn{8}{|l|}{ Weight — z score } \\
\hline Median & -2.1 & -2.7 & -2.6 & -1.9 & -2.8 & -2.7 & -2.6 \\
\hline 10th-90th percentile & -4.6 to 0.0 & -5.3 to -0.6 & -5.3 to -0.2 & -3.9 to -0.5 & -5.2 to -0.7 & -5.2 to -0.5 & -5.2 to -0.4 \\
\hline \multicolumn{8}{|l|}{ Height — z score } \\
\hline Median & -1.8 & -2.5 & -2.3 & -1.6 & -2.5 & -2.3 & -2.3 \\
\hline 10th-90th percentile & -3.2 to 0.1 & -4.0 to -0.8 & -4.0 to -0.4 & -3.7 to 0.2 & -4.2 to -0.8 & -4.0 to -0.3 & -4.0 to -0.4 \\
\hline \multicolumn{8}{|l|}{ CD4+ T cells - \% } \\
\hline Median & 16.0 & 14.0 & 14.9 & 18.9 & 14.4 & 15.0 & 15.0 \\
\hline 10th-90th percentile & 11.0 to 28.0 & 8.1 to 23.0 & 8.8 to 24.0 & 9.0 to 29.5 & 7.0 to 24.0 & 7.9 to 26.1 & 8.0 to 25.2 \\
\hline \multicolumn{8}{|l|}{ Plasma HIV-1 RNA — $\log _{10}$ copies/ml* } \\
\hline Median & 5.9 & 5.6 & 5.7 & 5.9 & 5.7 & 5.8 & 5.7 \\
\hline 10th-90th percentile & 5.0 to 5.9 & 4.8 to 5.9 & 4.9 to 5.9 & 4.8 to 5.9 & 4.6 to 5.9 & 4.7 to 5.9 & 4.8 to 5.9 \\
\hline WHO stage III or IV — no. (\%) & $21(51.2)$ & $71(67.0)$ & $92(62.6)$ & $19(52.8)$ & $69(66.3)$ & $88(62.9)$ & $180(62.7)$ \\
\hline $\begin{array}{l}\text { Documentation of no prior exposure to } \\
\text { nevirapine based only on oral } \\
\text { report - no. (\%) } \ddagger\end{array}$ & $10(24.4)$ & $9(8.5)$ & 19 (12.9) & $5(13.9)$ & $17(16.3)$ & $22(15.7)$ & $41(14.3)$ \\
\hline
\end{tabular}

* Data for HIV-1 RNA counts were censored at the upper limit of the assay (5.9 $\log _{10}$ copies per milliliter).

$\uparrow$ WHO denotes World Health Organization.

$\downarrow$ For all other infants, additional forms of documentation were available showing that they had not previously been exposed to nevirapine.

\section{PRIMARY END POINT}

The percentage of children who reached the primary end point (i.e., virologic failure or discontinuation of the study treatment for any reason, including death, by 24 weeks) was significantly higher in the nevirapine group than in the ritonavir-boosted lopinavir group (40.8\%, vs. $19.3 \%$; $\mathrm{P}<0.001$ ) (Table 2). The relative contributions of these end points were similar in the two treatment groups: $55.6 \%$ of the children in the ritonavir-boosted lopinavir group who reached the primary end point discontinued treatment before virologic failure, as compared with $45.0 \%$ in the nevirapine group (Table S2 in the Supplementary Appendix). The findings were similar across age strata (between-group difference in the percentage of children who reached the primary end point, 21.5 percentage points across both age strata, 22.0 percentage points for children younger than
12 months of age, and 21.3 percentage points for those 12 months of age or older) (Table 2 and Fig. 1A and 1B).

The results of primary end point analyses adjusted for age, sex, severity of HIV disease (based on WHO stage, percentage of CD4+ T cells, and HIV-1 RNA level), nevirapine dose at entry, documentation of prior single-dose nevirapine exposure (to prevent mother-to-child transmission), and HIV subtype were similar to the results of unadjusted analyses; only baseline HIV-1 RNA level was a significant predictor of the primary end point after adjustment for treatment $(P=0.03)$. Tests for interaction showed no evidence that the difference between treatment groups in the primary end point varied across subgroups $(\mathrm{P}=0.31$ for age, $\mathrm{P}=0.18$ for nevirapine dose, and $\mathrm{P} \geq 0.10$ for all other subgroups). 


\begin{tabular}{|c|c|c|c|c|c|c|}
\hline \multirow[t]{3}{*}{ Variable } & \multicolumn{2}{|c|}{ Nevirapine } & \multicolumn{2}{|c|}{$\begin{array}{l}\text { Ritonavir-Boosted } \\
\text { Lopinavir Group }\end{array}$} & \multirow[t]{2}{*}{$\begin{array}{l}\text { Between-Group } \\
\text { Difference } \\
(95 \% \mathrm{Cl}) \grave{\uparrow}\end{array}$} & \multirow[t]{3}{*}{ P Value } \\
\hline & All Children & $\begin{array}{l}\text { Rate of } \\
\text { End Point }\end{array}$ & All Children & $\begin{array}{l}\text { Rate of } \\
\text { End Point }\end{array}$ & & \\
\hline & no. & $\%$ & no. & $\%$ & percentage points & \\
\hline \multicolumn{7}{|l|}{ Age } \\
\hline$<12$ mo & 41 & 41.5 & 36 & 19.4 & $22.0(2.2-41.9)$ & 0.03 \\
\hline$\geq 12 \mathrm{mo}$ & 106 & 40.6 & 104 & 19.2 & $21.3(9.3-33.4)$ & 0.001 \\
\hline \multicolumn{7}{|l|}{ All children } \\
\hline Unweighted analysis & 147 & 40.8 & 140 & 19.3 & $21.5(11.2-31.8)$ & $<0.001$ \\
\hline Weighted analysis & 147 & 40.8 & 140 & 19.3 & $21.5(11.2-31.8)$ & $<0.001$ \\
\hline
\end{tabular}

* The rates of the primary end points (virologic failure or treatment discontinuation by study week 24) were estimated with the use of Kaplan-Meier methods. The weighted analysis was the primary study comparison; in the unweighted analysis, children were not stratified according to age. $\mathrm{Cl}$ denotes confidence interval.

$\uparrow$ The between-group difference is the rate in the nevirapine group minus the rate in the ritonavir-boosted lopinavir group.

\section{SECONDARY ANALYSES}

Rates of virologic failure or death by week 24 were 15.6 percentage points higher in the nevirapine group than in the ritonavir-boosted lopinavir group (95\% confidence interval [CI], 6.3 to 24.8; $\mathrm{P}=0.001$ ) (Fig. 1C and 1D). The hazard ratio for time to virologic failure or death by week 24 for children in the nevirapine group, as compared with those in the ritonavir-boosted lopinavir group, was 2.51 (95\% CI, 1.41 to 4.47; $\mathrm{P}<0.01$ ); adjusted analyses had similar results. Analyses of time to virologic failure or discontinuation of study treatment and time to virologic failure or death during the follow-up period significantly favored the ritonavir-boosted lopinavir group (Fig. 1). For all study end points, the difference between the treatment groups had been largely established by 24 weeks. By 48 weeks, 81 of 108 children $(75.0 \%)$ randomly assigned to nevirapine had HIV-1 RNA levels below 400 copies per milliliter, as compared with 101 of 119 children $(84.9 \%)$ randomly assigned to ritonavir-boosted lopinavir $(\mathrm{P}=0.06)$.

At study entry, 58 of 287 mothers (20.2\%) were breast-feeding their infants $(18.4 \%$ of the mothers of infants in the nevirapine group and $22.1 \%$ of those in the ritonavir-boosted lopinavir group), and none of the mothers were receiving ART. During study follow-up, mothers of 6 infants in the nevirapine group and 5 infants in the ritona- vir-boosted lopinavir group started ART, which in all cases included nevirapine. Only one of these children (in the ritonavir-boosted lopinavir group) had virologic failure, making it unlikely that maternal use of nevirapine-based ART influenced the study results. Multiple analyses showed that the initial nevirapine dose had no effect on hazard ratios for any end point or toxicity outcome.

\section{RESISTANCE TO ANTIRETROVIRAL AGENTS}

Accurate assignment to cohort 2 was dependent on the absence of prior maternal or infant exposure to NNRTIs. The results of baseline testing for resistance to antiretroviral agents (ViroSeq HIV-1 Genotyping System for population sequencing [Celera]) supported the assignments. Only 5 of 257 children $(2.0 \%)$ with samples that could be evaluated had mutations conferring nevirapine resistance (Y181C in 4 and $\mathrm{K} 103 \mathrm{~N}$ in 1) at baseline; only 1 of these 5 children was randomly assigned to the nevirapine group.

Among the 45 children in the nevirapine group who had virologic failure, resistance data were available at the time of failure for 32 children (for 23 by the visit at 24 weeks). At the time of virologic failure, 11 children had no detectable resistance, 17 had resistance to both nucleoside reverse-transcriptase inhibitors (NRTIs) and NNRTIs, 2 had resistance only to NRTIs, and 


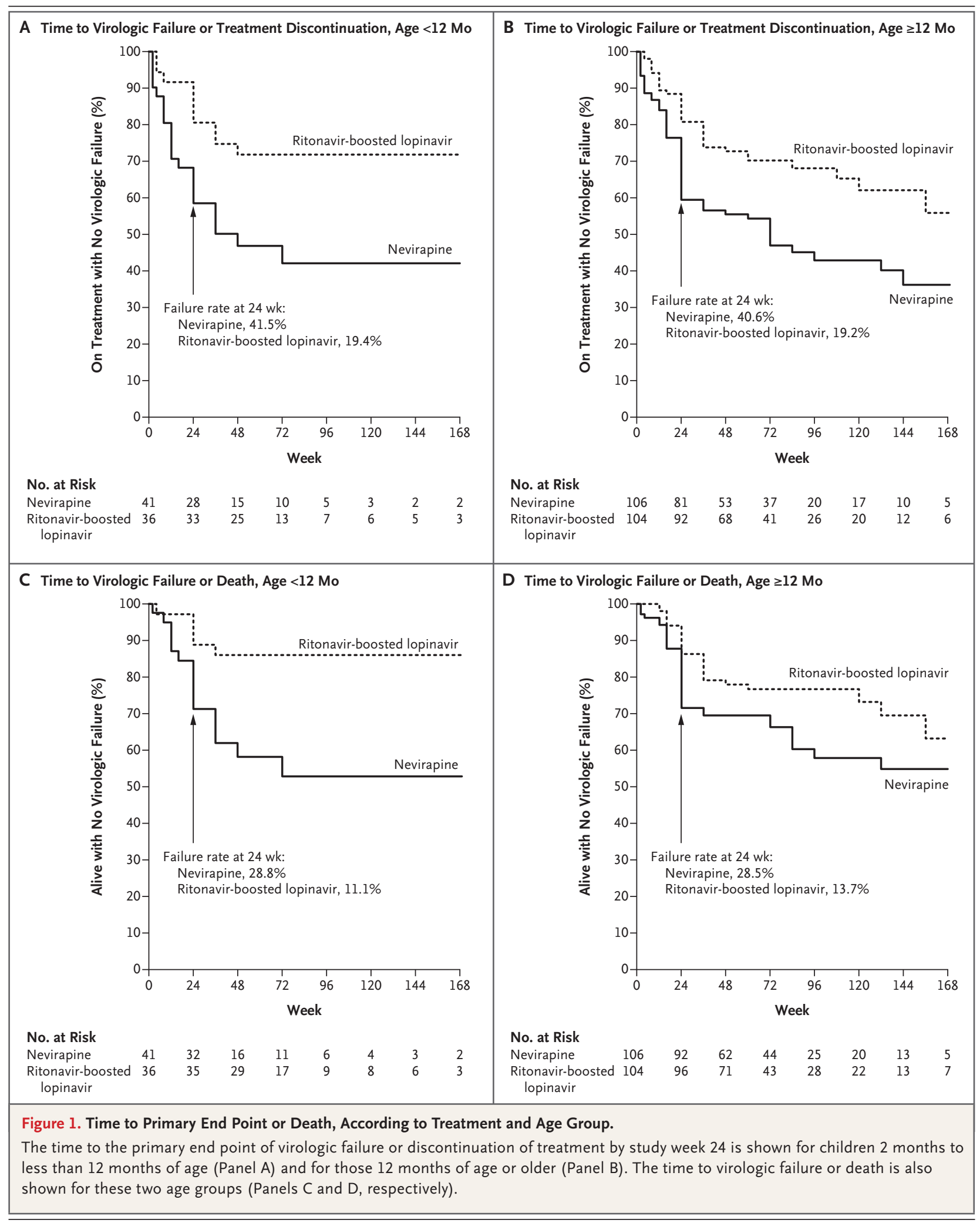

The New England Journal of Medicine 


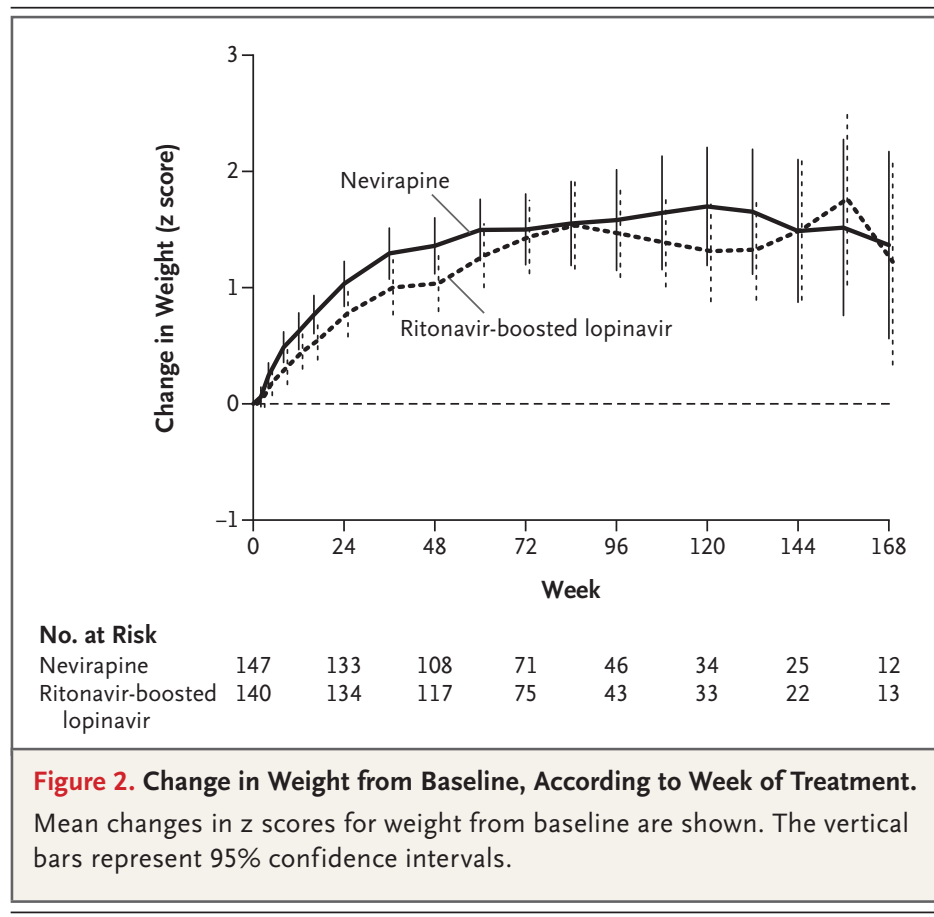

2 had resistance only to NNRTIs. Among children in the ritonavir-boosted lopinavir group, resistance data were available for 20 of 29 children with virologic failure at the time of failure: 9 had no detectable resistance, and 11 had resistance to NRTIs (1 of whom also had resistance to nelfinavir).

\section{SAFETY ANALYSIS AND ADVERSE EVENTS}

There were 13 deaths in the study: 10 in the nevirapine group and 3 in the ritonavir-boosted lopinavir group (hazard ratio with nevirapine, 3.41; $95 \%$ CI, 0.94 to $12.40 ; \mathrm{P}=0.06$ by the Wald test; $\mathrm{P}=0.05$ by exact log-rank test). Seven of the deaths in the nevirapine group occurred within the first 12 weeks of the study (Fig. S1 in the Supplementary Appendix). In 9 children, the primary cause of death was infection: gastroenteritis and diarrhea with marasmus (in 4 children), pneumonia (in 2), sepsis associated with burns and kwashiorkor (in 2), and malaria (in 1); the cause of death was unknown in 1 child. Among the 3 deaths in children receiving ritonavirboosted lopinavir, 1 was due to measles, 1 to pneumonia and cardiac failure, and 1 to gastroenteritis and marasmus.

Protocol-defined toxicity end points occurred in 19 children: 14 in the nevirapine group and 5 in the ritonavir-boosted lopinavir group (Table S3 in the Supplementary Appendix). The time to a protocol-defined toxicity end point was shorter in the nevirapine group than in the ritonavirboosted lopinavir group (hazard ratio, 3.00; 95\% CI, 1.06 to $8.21 ; \mathrm{P}=0.04)$. The primary differences between treatment groups were higher rates of dermatologic and hepatic toxicity end points associated with nevirapine treatment (Tables S3 and S4 in the Supplementary Appendix). Changes in cholesterol and triglyceride levels at 24 and 48 weeks, adjusted for values at entry, did not differ significantly between the two treatment groups.

\section{IMMUNOLOGIC AND GROWTH RESPONSES}

Changes in the CD4+ count and percentage, weight and height $\mathrm{z}$ scores, and BMI from baseline to week 24 and to week 48 were compared according to treatment, with and without adjustment for baseline value and age stratum. Mean values improved for all outcomes in both treatment groups; between-group differences were significant only for adjusted changes in the $\mathrm{z}$ score for weight $(\mathrm{P}=0.01$ at week 24 and $\mathrm{P}=0.01$ at week 48) and BMI ( $\mathrm{P}=0.02$ at week 24 and $\mathrm{P}=0.03$ at week 48) and favored the nevirapine group (Fig. 2, and Table S5 in the Supplementary Appendix).

\section{DISCUSSION}

The results of this randomized clinical trial, which compared nevirapine-based ART and ritonavir-boosted lopinavir-based ART for the initial treatment of HIV-infected children, challenge our current approach. For infants and young children, regardless of whether they were previously exposed to nevirapine, we now have evidence of the superiority of ritonavir-boosted lopinavirbased regimens over nevirapine-based regimens in terms of both efficacy and safety.

Nevirapine-based treatment is currently the choice for first-line ART in most countries where resources are limited and is often the only readily available option. Recent data in HIV-infected women have provided support for the continued use of nevirapine as a first-line treatment option. The Optimal Combination Therapy after Nevirapine Exposure (OCTANE) A5208 trial, which had a design parallel to that of the P1060 study, showed that nevirapine, administered as the initial treatment in HIV-infected women without prior exposure to single-dose nevirapine, was noninferior to ritonavir-boosted lopinavir with respect to virologic failure or death ${ }^{2}$; however, nevirapine was associated with a poorer safety 
profile. The treatment regimen included tenofovir and emtricitabine, which may be more potent than zidovudine and lamivudine, the agents used in the P1060 study. In addition, the PENPACT 1 (Paediatric European Network for Treatment of AIDS [PENTA 9]/Pediatric AIDS Clinical Trials Network [PACTG] 390) trial, conducted in Europe, the United States, and South America, showed equivalent outcomes when treatment was initiated with an NNRTI-based regimen or a proteaseinhibitor-based regimen. ${ }^{4}$ The PENPACT 1 trial differed substantially from the P1060 trial in several respects: it enrolled children at a median age of 6.5 years, with a median viral load of 5.1 $\log _{10}$ copies per milliliter; the primary end point was virologic only (without consideration of treatment changes); and it allowed investigators to choose the NNRTIs (efavirenz was chosen in $62 \%$ of the cases) and protease inhibitors (which were equally divided between nelfinavir and ritonavir-boosted lopinavir). The P1060 study directly compared nevirapine-based treatment and ritonavir-boosted lopinavir-based treatment in children who were younger than 3 years of age.

Why did nevirapine fare less well than ritonavirboosted lopinavir among the infants in our study? One important factor may be the high plasma HIV-1 RNA levels commonly seen during infancy (median for the overall cohort, 536,000 copies per milliliter), which make viral suppression difficult. Even with optimal ART viral decay kinetics, the time to achievement of an undetectable plasma virus level will be longer for infants than for older children and adults, who have lower viral loads. ${ }^{5,6}$ It is plausible that the use of agents for which single-gene mutations result in resistance (e.g., nevirapine) may be suboptimal in the presence of high viral replication and a prolonged time to viral suppression, which may confer a predisposition to the emergence of resistance. In the P1060 study, more than half the children in the nevirapine group who had virologic failure had nevirapine resistance at the time of failure. The results of the PENPACT 1 study showed that NNRTI resistance in children with no response to NNRTI-based ART occurs early and at low levels of viral rebound $(<1000$ copies per milliliter). ${ }^{4}$

Another potential contributing factor may have been the ramp-up dosing strategy, in which nevirapine is given once daily at half the final target dose for 2 weeks and is then increased to the full dose on a twice-daily schedule. This strategy was designed to minimize the risk of dermatologic reactions during the initiation of nevirapine therapy (seen primarily in adults during drug development) and to accommodate the induction of cytochrome P-450 metabolizing enzymes after exposure to nevirapine. However, this may result in suboptimal levels of nevirapine during the ramp-up period, a time when viral levels in infected infants are profoundly elevated. This hypothesis is consistent with the finding that virologic failure in the nevirapine group tended to be an early event, occurring in the first 12 to 24 weeks of therapy. The Children with Human Immunodeficiency Virus (HIV) in Africa - Pharmacokinetics and Adherence of Simple Antiretroviral Regimens (CHAPAS 1) study compared the initiation of full-dose nevirapine in fixed-dose combination tablets to simplify administration in children with a median age of 5 years. ${ }^{7}$ Rash occurred at a higher frequency when nevirapine was initiated at the full dose ( $11 \%$, vs. $2 \%$ with dose escalation) but was considered to be manageable.

Baseline resistance to NNRTIs due to unrecognized exposure to drugs for preventing motherto-child transmission in cohort 2 is not likely to be responsible for the results; the frequency of baseline nevirapine resistance was less than $1 \%$ ( 1 of 132 children) in the nevirapine group. Resistance was determined with the use of an HIVgenotyping system based on population sequencing and approved by the Food and Drug Administration. Low-frequency NNRTI mutations, which can be detected only by means of highsensitivity assays, have been implicated in treatment failure in adults. ${ }^{8,9}$ It is not known whether low-frequency mutations at baseline affected treatment outcomes in the P1060 cohort; this question will require further research. However, in the Nevirapine Resistance Study (NEVEREST), virologic failure in infants who were switched to nevirapine-based ART was associated with an increased frequency of baseline resistance, as detected by population sequencing, but not with low-frequency baseline resistance. ${ }^{10}$

Data from P1060 cohort 1 (infants exposed to single-dose nevirapine) ${ }^{1}$ and from NEVEREST ${ }^{11}$ previously showed that CD4 counts and weight gain improved more slowly from baseline levels with ritonavir-boosted lopinavir than with nevirapine despite efficacy data favoring ritonavirboosted lopinavir. Although somewhat counterintuitive, this observation was partially confirmed 
in the present study, in which adjusted increases in the $\mathrm{z}$ score for weight and in BMI from baseline to week 24 and to week 48 were significantly larger in the nevirapine-treated group; however, changes in CD4+ counts over time appeared to be similar in the two groups. The mechanism underlying the smaller weight gain in the ritonavir-boosted lopinavir group remains unclear, but leading hypotheses are poor palatability and appetite suppression, as well as the metabolic consequences of ritonavir, excipients, or both in the liquid formulation (15.3\% propylene glycol and $42.4 \%$ alcohol) or both. ${ }^{1,12-15}$

The path forward for pediatric first-line treatment guidelines in resource-limited settings is not simple. Identification of HIV infection early in infancy, with prompt initiation of ART, is critical for increasing survival among HIV-infected children. ${ }^{3,16}$ The data from the P1060 cohort 2 study show superior outcomes with ritonavirboosted lopinavir, as compared with nevirapine, for the composite end point, as well as for virologic failure or death, toxicity, and death alone. These data support ritonavir-boosted lopinavir as the basis for first-line ART in all children younger than 3 years of age, regardless of whether they have had prior NNRTI exposure. Enthusiasm for such an approach, however, may be tempered by the inherent challenges to its implementation worldwide. For example, the liquid formulation of ritonavir-boosted lopinavir has an unpleasant taste and does not withstand high ambient temperatures. Today, the cost of a ritonavir-boosted lopinavir-based treatment regimen for young children is approximately twice that of a nevirapine-based regimen. It is important to note that $75 \%$ of the children who were randomly assigned to nevirapine had HIV-1 RNA levels below 400 copies per milliliter after 48 weeks of therapy, although the percentage was higher for the children randomly assigned to ritonavir-boosted lopinavir. In the absence of a confirmatory study, policymakers are left to weigh the costs and benefits of these two different first-line regimens as they develop national and regional pediatric treatment guidelines. New approaches to monitoring treatment and new drug formulations are urgently needed to address this emerging gap in global pediatric HIV treatment.

The views expressed are those of the authors and do not necessarily represent the official policies of the Department of Health and Human Services, and the mention of any trade names, commercial packages, or organizations does not imply endorsement by the U.S. government.

Supported by a grant [U01 AI068632] from the National Institute of Allergy and Infectious Diseases (NIAID), the Eunice Kennedy Shriver National Institute of Child Health and Human Development, and the National Institute of Mental Health to the Pediatric AIDS Clinical Trials Group (PACTG) and the International Maternal Pediatric Adolescent AIDS Clinical Trials (IMPAACT) Group; the Statistical and Data Analysis Center at Harvard School of Public Health under NIAID cooperative agreements (5 U01 AI41110 with the PACTG and 1 U01 AI068616 and U01 AI068632 with the IMPAACT Group); and by a contract from the NIAID (HHSN272200800014C).

Dr. Avy Violari reports receiving lecture fees from Abbott, consulting fees from Tibotec, and trial drugs from GlaxoSmithKline; Dr. Hughes, consulting fees from Boehringer Ingelheim, Pfizer, Medicines Development, and Tibotec and support for a scientific workshop from Merck; Dr. Cotton, lecture fees from Abbott; and Dr. Moultrie, grants from GlaxoSmithKline, ViiV Healthcare, and Tibotec. No other potential conflict of interest relevant to this article was reported.

Disclosure forms provided by the authors are available with the full text of this article at NEJM.org.

We thank the children, their families, and the caregivers who agreed to participate in P1060 and who placed their trust in the site clinical study teams; P1060 study team members Joan Coetzee, Emily Barr, Phillippa Musoke, Mutsawashe Bwakura-Dangarembizi, Tammy Meyers, Robert Bollinger, George Kafulafula, Namwinga Chintu, Tamara Kuryla, Carrie Fry, and Don Decker, as well as Sandi Lehrman, for their contributions to the study; and Lauren Petrella and Peter Piliero of Boehringer Ingelheim, Dr. Marisol Martinez of Abbott, and Navdeep Thoofer and Wendy Snowden of GlaxoSmithKline for their provision of the antiretroviral agents used in the study.

\section{APPENDIX}

The authors' affiliations are as follows: Perinatal HIV Research Unit, University of the Witwatersrand, Johannesburg (A.V.); the University of the Witwatersrand Reproductive Health and HIV Institute, Faculty of Health Sciences, Johannesburg (H.M.); Tygerberg Children's Hospital, Stellenbosch University, Tygerberg (M.F.C.); and the Nelson R Mandela School of Medicine, University of KwaZulu-Natal, Durban (R.B.) - all in South Africa; Harvard School of Public Health, Boston (J.C.L., M.D.H.); the Department of Paediatrics and Child Health, University of Zimbabwe College of Health Sciences, Harare, Zimbabwe (H.A.M.); Makerere University-Johns Hopkins University Research Collaboration, Kampala, Uganda (L.B.-M.); University of North Carolina Project, Lilongwe, Malawi (P.K.); the Center for Infectious Disease Research in Zambia, Lusaka, Zambia (B.H.C.); the Department of Pediatrics, B.J. Medical College and Sassoon General Hospital, Pune, India (S.K.); Duke University-Kilimanjaro Christian Medical Center Collaboration, Moshi, Tanzania (W.S.); Pharmaceutical Affairs Branch (L.P.) and the Henry Jackson Foundation (P.J.-P.), the Division of AIDS, National Institute of Allergy and Infectious Diseases, National Institutes of Health, Bethesda; Social and Scientific Systems, Silver Spring (E.P.); the Pediatric, Adolescent, and Maternal AIDS Branch, Eunice Kennedy Shriver National Institute of Child Health and Human Development, National Institutes of Health, Rockville (L.M.M.); and Johns Hopkins University School of Medicine, Baltimore (S.H.E.) - all in Maryland; International Center for AIDS Care and Treatment Programs, Columbia University Mailman School of Public Health and College of Physicians and Surgeons, New York (E.J.A.); and Frontier Science and Technology Research Foundation, Amherst (L.M.) — both in New York; and Dartmouth Medical School, Lebanon, NH (P.P.). 
REFERENCES

1. Palumbo P, Lindsey JC, Hughes MD, et al. Antiretroviral treatment for children with peripartum nevirapine exposure. N Engl J Med 2010;363:1510-20.

2. Lockman S, Hughes MD, McIntyre J, et al. Antiretroviral therapies in women after single-dose nevirapine exposure. N Engl J Med 2010;363:1499-509.

3. Antiretroviral therapy of HIV infection in infants and children: towards universal access: recommendations for a public health approach -2010 revision. Geneva: World Health Organization Technical Reference Group, 2010.

4. Babiker A, Castro HG, Compagnucci A, et al. First-line antiretroviral therapy with a protease inhibitor versus nonnucleoside reverse transcriptase inhibitor and switch at higher versus low viral load in HIV-infected children: an open-label, randomized phase 2/3 trial. Lancet Infect Dis 2011;11:273-83.

5. Palumbo P, Wu H, Chadwick E, et al. Virologic response to potent antiretroviral therapy and modeling of HIV dynamics in early pediatric infection. J Infect Dis 2007;196:23-9.

6. Luzuriaga $\mathrm{K}, \mathrm{Wu} \mathrm{H}, \mathrm{McManus} \mathrm{M}$, et al. Dynamics of human immunodeficien- cy virus type 1 replication in vertically infected infants. J Virol 1999;73:362-7. 7. Mulenga V, Cook A, Walker AS, et al. Strategies for nevirapine initiation in HIV-infected children taking pediatric fixed-dose combination "baby pills" in Zambia: a randomized controlled trial. Clin Infect Dis 2010;51:1081-9.

8. Li JZ, Paredes R, Ribaudo HJ, et al. Low-frequency HIV-1 drug resistance mutations and risk of NNRTI-based antiretroviral treatment failure: a systematic review and pooled analysis. JAMA 2011; 305:1327-35.

9. Boltz VF, Zheng Y, Lockman S, et al. Role of low-frequency HIV-1 variants in failure of nevirapine-containing antiviral therapy in women previously exposed to single-dose nevirapine. Proc Natl Acad Sci U S A 2011;108:9202-7.

10. Hunt GM, Coovadia A, Abrams EJ, et al. HIV-1 drug resistance at antiretroviral treatment initiation in children previously exposed to single-dose nevirapine. AIDS 2011;25:1461-9.

11. Coovadia A, Abrams EJ, Stehlau R, et al. Reuse of nevirapine in exposed HIVinfected children after protease inhibitorbased viral suppression: a randomized controlled trial. JAMA 2010;304:108290. 12. Nachman SA, Lindsey JC, Pelton S, et al. Growth in human immunodeficiency virus-infected children receiving ritonavir-containing antiretroviral therapy. Arch Pediatr Adolesc Med 2002;156:497503.

13. Dreimane D, Nielsen K, Deveikis A, Bryson YJ, Geffner ME. Effect of protease inhibitors combined with standard antiretroviral therapy on linear growth and weight gain in human immunodeficiency virus type 1-infected children. Pediatr Infect Dis J 2001;20:315-6.

14. Buchacz K, Cervia JS, Lindsey JC, et al. Impact of protease inhibitor-containing combination antiretroviral therapies on height and weight growth in HIV-infected children. Pediatrics 2001;108(4):E72.

15. Aldrovandi GM, Lindsey JC, Jacobson DL, et al. Morphologic and metabolic abnormalities in vertically HIV-infected children and youth. AIDS 2009;23:661-72. 16. Violari A, Cotton MF, Gibb DM, et al. Early antiretroviral therapy and mortality among HIV-infected infants. N Engl J Med 2008;359:2233-44.

Copyright () 2012 Massachusetts Medical Society. and browsing of titles and tables of contents is easy and available to all.

Individual subscribers are entitled to free 24-hour access to 50 archive articles per year. Access to content in the archive is available on a per-article basis and is also being provided through many institutional subscriptions. 